\title{
Sub Mucous Cleft Palate and Its Diagnosis and Management
}

\author{
Pradhan $\mathbf{B}^{1}$
}

${ }^{1}$ Bhawani Pradhan, Speech Pathology \& Audiologist, Shree Birendra Hospital, Chhauni

\begin{abstract}
Four different age groups of cases came to hospital with the complaint of severely nasalized speech and since childhood. Parents had already consulted to the physician in one of the valley Hospital who referred the patient for speech therapy without a clinical diagnosed as sub mucous cleft (SMC). Speech pathologist has to confirm of sub mucous cleft palate with the help of the plastic surgeon.

Sub mucous cleft palate is a significant cause of severs speech and language disorder. Aim of the paper is to make the clinician and laymen aware of the sub mucous cleft palate which is less commonly diagnosed partly due to ignorance and misdiagnosis.

Sub mucous cleft is the cleft of muscle and bony portion of palate it has got triad characters:- Bifid uvular, thin bluish line in the margin raphe (septum palludine) \& hyper nasality. And we could feel palpable sign and speech and language disorder. It may have also lots of related problems like mental retarted, hearing loss, behavior problems etc.

Sub mucous cleft, is diagnosed and management by team approach like plastic surgeon, E.N.T. Surgeon, Dental surgeon, sp \& lag pathologist and audiologist etc. Speech therapy is the one of the essential area for their better quality of life after surgery.
\end{abstract}

\section{Introduction}

Sub mucous cleft palate is characterized by triad characters:- Bifid uvular, thin bluish line in the margin raphe (septum palludine) \& hyper nasality and also speech and language ${ }^{1}$.

S.M.C is generally confused \& ignored by clinician \& parents until they prominently shown their speech \& language problem. Some of them need to have surgery \& speech therapy and some them need only speech therapy. They can be diagnosed \& managed by team approach speech therapy is one of most essential area for their better quality of life after surgery.

\section{Clinical Feature of SMC}

Anatomically they must have got bifid uvular, thin bluish line in mid line soft palate, also seen bony notch in hard palate, attenuation of mid line raphe ${ }^{1}$. And we could also see in function abnormalities like palpable

sigh in hard palate, nasalized speech \& audible nasal air emission too. Most important is speech \& language disorder.

\section{Case Report}

Four different age group of cases(w-7/m, x-8y/f, $y-10 y / m$ \& $z-13 y / f)$ came with the complaint of speech $\&$ language defect since childhood. Some of them also complaining about car discharge \& diminished hearing. Some of then having positive family history some of them not. They were found that hard palate shown to be normal but soft palate shown to be normal but soft palate shown thin bluish line in middle \& bifid uvula. Their voice \& respiration was normal but resonance was hyper \& defect of articulation.

All of them consulted to different hospital for about their speech problems. All the physician directly refer 
to speech therapy without diagnosis. Lastly they were conform diagnosis by plastic surgeon. After that they went under surgery. Then speech therapy was taken. This shows markedly improvement by taking speech therapy.

\section{Discussion}

Calnan (1954) has given triad sign of sub Muscous cleft palate that is Bony notch in hard palate, thin bluish line in Mid line of Soft palate and Bifid Uvula which is easy to recognized about the sub mucous cleft palate ${ }^{1}$.

Pennbeker (1984) has done 25 years of research about language ability of cleft palate which was found that language ability of this child tend to reduced due to hearing loss, lacks of exposure in outer environments and introverts nature and frequent visited in hospitals etc ${ }^{2}$. Trost-Cord Manes (1990)- found that cleft palate children tend to have velopharyngeal incompetence (V.P.I.) which leads to inability to sustain intra oral air pressure which can't balance between oral \& nasal air flow during speech ${ }^{3}$. And also affects for feeding due to difficult to produce negative pressure during feeding. Mc William (1993) found that cleft palate children tend to have low language ability due to tensor palatine muscles defect which leads to Otitis media and hearing loss, because of hearing loss children tend to have low language ability ${ }^{4}$.

\section{Related Disorder}

Feeding problems: This problemsareverycommon in infant stage of sub mucous cleft children. (W.B.jones) Among them are choking, excessive are intake requiring several burping, inability to create sufficient suction to pull milk from nipple, Nasal reguargiation and slowness to complete feeding (Arvedson 1992) ${ }^{5}$,

(all 4 cases found that nasal regurgitation)

E.N.T and hearing problems: Because of otitis media occurs more frequently in individual with sub mucous cleft need consistent E.N.T. check up ${ }^{6}$. Hearing loss is frequently common for these cases so Audio logical consultation is also critical. (2 cases found that otitis media \& mild hearing loss and 2 cases found that normal ear condition)

Dental and Orthodontic: Complication related to cleft cases is also common so it required careful coordination between orthodontic and surgical management of lip, palate and alveolar ridge ${ }^{5}$. (All cases found that dental problems)
Psychological Problems: It may occur among children, teems and adult with sub mucous cleft as they face the same challgenge in life as anyone else, plus some challenge unique to their condition, there were some people who adopt well to their condition they will survive as normal life with other hand who developed low self esteem and dissatisfaction with their communication defects ${ }^{7}$. (2 younger cases shown to be normal psychological condition and social adjustment but rest two adolescent shown to be little bit shy and introvert nature \& didn't want to interact with other peer group.)

\section{Speech and language problems}

Sub mucous cleft children are prone to have speech and language inadequacy and severe problems Delay Speech. Language acquired is quite common for these children for 3-4 years. Some children we finds language deviant, however their language is normal still their speech characters are very effective ${ }^{7}$. They tend to have hoarseness of voice due to excessive pressure in larynx. Faulty placement of articulators is very common so they will have severe misarticulation duet to faulty learning \& compensation articulation. And most common \& remarkably find hyper resonance speech which their speech heard as nasalized speech. (all of them found to be nasalized speech and severe articulation defect.)

\section{Speech \& Language Disorder}

Velopharyngeal Dysfunction: Includes hyper nasal resonance and nasal distortion of pressure consonant caused by inability to sustain and control of intra oral air pressure the result in inadequate balance of oral and nasal air pressure during speaking.

Velopharyngeral dysfunctions of these children are main caused by structure defect ${ }^{8}$.

Phonation- Resonance A lack of coordination in the timing of respiratory, larynges, articulators and Velopharyngeral system may give perception of hyper nasality and slow the speaking rate in speakers with Velopharyngeral incompetence ${ }^{8}$. Other symptom that may develop either from inadequacy control of air pressure due to Velopharyngeral dysfunction or from compensatory strategies, include in pitch range use of a soft aspirate voice, monotonous pitch and strangled and hoarseness of bvoice and extremely loud and effortful phonation. (All of them have hyper resonance and hoarseness of voice) 
Articulation problems Either due to structural defect or faulty learning if they are repairing al late age they tend to have articulation disorder. Substitution of articulation and glottal stop, Distortion and Omission are very common for these $\operatorname{cases}^{9}$ (all of them have severe matriculation like glottal stop, substation of sound etc.)

Language Disorder Although not as common as speech disorder delay early language development is possible, Children with sub mucous cleft. Generally present normal receptive language development severe factor hover may cause with sub mucous cleft shows poor expression ${ }^{7}$ (all of them have normal language level).

\section{Diagnosis}

The TEAM approach to evaluating and treating cleft palate is most important. There is interdisciplinary and transdisciplinery is more common, in this team includes surgeon, Pediatrician, Dietician, Dentist, Psychiatrics \& Psychologist, Speech and language pathologist \& Audiologist and Parents, school teachers, social workers etc ${ }^{10}$. All team members are equally Responsible for evaluating and treatment for cleft palate and sub mucous children ${ }^{11}$.

Speech and Language pathologist: is responsible for monitoring and screening all areas of speech and language in clients with cleft lip/palate and sub mucous cleft. Ealuating procedure may be classified as screening to determine the need for in depth evaluation, Diagnosis evaluation and programming evaluation to identify therapy objectives, effective therapy procedure and the probable prognosis and program assessment to determine effectiveness of treatment, speech and language pathologist may be the recognize the possible syndromes $^{12}$.

Other client and family plays vital roles in the Team decision making process and including the family as team members. Parents and client education about cleft palate is an important components of the treatment process.

\section{Treatment}

All Team members are responsible for their treatment and management. They does their treatments respectably to their areas.

Speech and language pathologist provides treatment for language delay development, resonance problems, articulation disorder and compensatory articulation like glottal stop and hoarseness of voice ${ }^{1}$.

Children with sub mucous cleft are at high risk for developing speech and language disorder or delay. Therefore preventing the development of language delay or compensatory articulation error such as glottal stop are high priorities during the preschool year. Parents and other primary care givers is primary provider of language stimulation for children with sub mucous cleft ${ }^{1}$. Clinician provides information and suggestion about language stimulation activities. If language delay of disorder found formal language therapy is identified, then parents and clinician should work as team. Family education about strategies for stimulation and languages development is an important aspect of comprehensive treatment for child with sub mucous cleft.

The parents, members of the health care team and school personnel's should cooperate is effort to build the child self esteem and self confident ${ }^{13}$, Because behaviors is an important variables in peer acceptance, they should set the behavior expectation for the child with sub mucous cleft as for any other child.

\section{Conclusion}

Sub mucous cleft(S.M.C) palate is seems to be less common may be due to improper diagnosis or over looked by physicians with or without speech problems. But S.M.C is the significant cause for speech $\&$ language problem \& most of them tend to have hyper resounance S.M.C. is manage by team approach like surgeon, pediatrician, Audiologist \& speech \& language pathologist and speech therapy is one of the essentials for their better quality of life after surgery.

\section{References}

1. Croft C.B. Shprintzen, R.J. Daniller. A. and Lewin, M.L. the occults sub mucous cleft palate and muscular Uvular, cleft palate Journal vol 15, No 2150-154 (1997).

2. Mc. Willeams, B.J. cleft palate, In shameer, G. and W, gG.E.)eds) communication disorders P.P.330369). Columbus, OH: Charles E. Mersell, 1982 b.

3. Pennbecker M. Language abilities of individuals with cleft palate: Implication for Intervention, paper presented at the annual meeting of the texas speech language hearing association, Hauston, Tx- February 1989. 
4. Seltor R.L. Hahn E. \& Morris H.L. Diagnosis \& therapy. In spriestesback, D.C. and Sherman, D(eds) cleft palate \& communication. Pp 225-268, New york, Academic press 1968.

5. Arvedson, J.C. Infant Oral - Motor function and feeding. In Brodsky, Hall, L. and ritter Schmida. D.H.(eds), cranio facial Anomalies, An interdisciplinary Approach, Pp. 188-195, Louis C.V. Morby.

6. Van Dr and Hardin. M.D speech therapy for the child with cleft lip \& Palate, In Bordach, J. and Morris H.L.(eds) Multidisciplinary management of cleft lip \& palate Pp 799-806. Philadelphia W.B. saunders company 1990.

7. Bevis R.C. Orthodontic diagnosis and treatment procedures, In moller, K.T and Star C.D.(Eds) cleft palate interdisciplinary Issues and treatment $\mathrm{Pp}$ 121-144. Austin Tx pro-Ed 1993.

8. Bluesstones C.D and Klein, J.O.otitis Media in infant and children, Philadelphia, P.P: WW.b.Saunders company 1988.
9. Borley, E.C. Technique, for Articulatory Disorders Spring field, II: charle C. Tomes, 1981.

10. Broen, P.P. Doyle, S.S. and Bacon, C.K. the velopharyngeally Inadequate child phonologic, change with intervention, cleft palate - caniofacial journal, vol 30, No 5:500-507 ( 1993).

11. Bzoch K.C (Eds) communication Disorder, Rrlated to cleft lip and palate Boston's college Hill 1981.

12. Golding - Kushner, K.J. Treatment and resonance disorders associated with cleft palate and V.P.I in shprintzen, R.J. and Bardah. J(Eds) cleft palate speech amangement. A multidisciplinary Approach p.p 327-351, St Lousis C.V. Morby, 1995.

13. Trost - cardamane, J.E. The development of speech: assessing cleft palate misarticulations. In Kernahan, D.A. and Rosenstein, Cleft lip and palate: A system of management, Pp. 227-235, Baltimore: Williams and Wilkins, 1990b. 